\title{
Integrability and dark states in an anisotropic central spin model
}

\author{
Tamiro Villazon $\odot,{ }^{1}$ Anushya Chandran, ${ }^{1}$ and Pieter W. Claeys $\odot^{2, *}$ \\ ${ }^{1}$ Department of Physics, Boston University, 590 Commonwealth Avenue, Boston, Massachusetts 02215, USA \\ ${ }^{2}$ TCM Group, Cavendish Laboratory, University of Cambridge, Cambridge CB3 OHE, United Kingdom
}

(Received 14 February 2020; revised 30 July 2020; accepted 12 August 2020; published 28 August 2020)

\begin{abstract}
Central spin models describe a variety of quantum systems in which a spin- $\frac{1}{2}$ qubit interacts with a bath of surrounding spins, as realized in quantum dots and defect centers in diamond. We show that the fully anisotropic central spin Hamiltonian with (XX) Heisenberg interactions is integrable. Building on the class of integrable Richardson-Gaudin models, we derive an extensive set of conserved quantities and obtain the exact eigenstates using the Bethe ansatz. These states divide into two exponentially large classes: bright states, where the qubit is entangled with the bath, and dark states, where it is not. We discuss how dark states limit qubit-assisted spin bath polarization and provide a robust long-lived quantum memory for qubit states.
\end{abstract}

DOI: 10.1103/PhysRevResearch.2.032052

Introduction. With the advent of new quantum technologies, there is increasing interest in using small quantum systems to control and coherently manipulate mesoscopic environments [1-6]. In the simplest setting, a single spin- $\frac{1}{2}$ qubit controls a surrounding bath of spins, extending the available degrees of freedom and turning the detrimental effects of the bath into a useful resource. These systems are modeled by central spin (or spin star) Hamiltonians, as schematically illustrated in Fig. 1. Central spin models have broad applicability in quantum information $[7,8]$, and quantum metrology and sensing $[9,10]$, and describe the interactions between nitrogen-vacancy centers and nuclear spins in diamond $[11,12]$ and the hyperfine interaction in quantum dots [13-15].

The central spin $\vec{S}_{0}$ typically interacts with the bath spins $\vec{S}_{i}$ through anisotropic Heisenberg interactions $\propto S_{0}^{x} S_{i}^{x}+S_{0}^{y} S_{i}^{y}+$ $\alpha S_{0}^{z} S_{i}^{z}$. The fully isotropic XXX model $(\alpha=1)$ is common in systems with emergent spherical symmetry, e.g., quantum dots in semiconductors with $s$-type conduction bands $[13,14]$, while the fully anisotropic XX model $(\alpha=0)$ arises in resonant dipolar spin systems in rotating frames [16-22]. Crucially, the fully isotropic XXX model is integrable, belonging to the class of XXX Richardson-Gaudin integrable models [23-25]. Integrability guarantees an extensive set of conserved quantities and allows all eigenstates to be exactly obtained using Bethe ansatz techniques, which has led to various studies of the equilibrium and dynamical properties of the XXX model [10,26-30]. However, generic central spin models with $\alpha \neq 1$ are not known to be integrable.

\footnotetext{
*pc652@cam.ac.uk
}

Published by the American Physical Society under the terms of the Creative Commons Attribution 4.0 International license. Further distribution of this work must maintain attribution to the author(s) and the published article's title, journal citation, and DOI.
In this Rapid Communication, we show that the fully anisotropic XX model $(\alpha=0)$ is integrable and exhibits a rich eigenstate structure. Its Hamiltonian describes a central spin- $\frac{1}{2}$ qubit in an external magnetic field $\omega_{0}$, interacting with a bath of $L$ spins in a uniform field $\omega$,

$$
H=\omega_{0} S_{0}^{z}+\omega \sum_{i=1}^{L} S_{i}^{z}+\sum_{i=1}^{L} g_{i}\left(S_{0}^{+} S_{i}^{-}+S_{0}^{-} S_{i}^{+}\right) .
$$

The interaction strengths $g_{i}$ are taken to be inhomogeneous and the bath spins can have arbitrary spin $s_{i}$ (see Fig. 1). Since $H$ conserves total spin projection $S^{z}=S_{0}^{z}+\sum_{i} S_{i}^{z}$, we set $\omega=0$ without loss of generality. To establish the integrability of $H$, we present an extensive number of conserved charges and construct the exact eigenstates using the Bethe ansatz [23]. We note that Jivulescu et al. previously used Bethe ansatz techniques to construct a subset of exact eigenstates [31,32], but did not show that $H$ is integrable.

Remarkably, the eigenstates of the XX model separate into two exponentially large classes with distinct entanglement structure. Dark states $|\mathcal{D}\rangle$ have a product state (unentangled) structure $|\downarrow\rangle_{0} \otimes\left|\mathcal{D}^{-}\right\rangle$or $|\uparrow\rangle_{0} \otimes\left|\mathcal{D}^{+}\right\rangle$, where the central spin is fully polarized along the $z$ direction and the bath state satisfies [33-37]

$$
\left(\sum_{i=1}^{L} g_{i} S_{i}^{ \pm}\right)\left|\mathcal{D}^{ \pm}\right\rangle=0 .
$$

Dark states are independent of $\omega_{0}$ and form degenerate manifolds with energy $\pm \omega_{0} / 2$ in every $S^{z}$ sector. In contrast, bright states $|\mathcal{B}\rangle$ exhibit qubit-bath entanglement and are given by linear combinations of definite central spin projection $c_{\downarrow}|\downarrow\rangle_{0} \otimes\left|\mathcal{B}^{-}\right\rangle+c_{\uparrow}|\uparrow\rangle_{0} \otimes\left|\mathcal{B}^{+}\right\rangle$. These states explicitly depend on $\omega_{0}$, and arise in pairs exhibiting level repulsion in the eigenspectrum of $H$ (schematically shown in Fig. 2).

Conserved charges. The conserved charges of the Hamiltonian $H$ in Eq. (1) follow from the class of integrable XXZ Richardson-Gaudin models [23-25] (see the explicit 


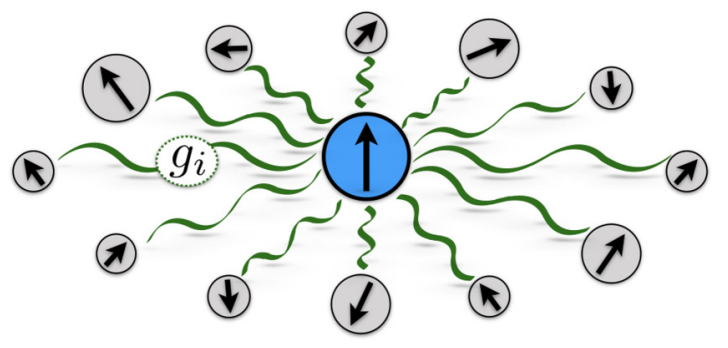

FIG. 1. Schematic representation of the central spin model. A central spin- $\frac{1}{2}$ particle interacts with an environment of $L$ spin- $s_{i}$ particles with interaction strengths $g_{i}, i=1 \ldots L$.

derivation below), and are given by

$$
\begin{aligned}
Q_{i}= & -2 S_{0}^{z} S_{i}^{z}+\frac{1}{2}\left(S_{i}^{+} S_{i}^{-}+S_{i}^{-} S_{i}^{+}\right) \\
& +\sum_{\substack{j=1 \\
j \neq i}}^{L}\left[\frac{g_{i} g_{j}}{g_{i}^{2}-g_{j}^{2}}\left(S_{i}^{+} S_{j}^{-}+S_{i}^{-} S_{j}^{+}\right)+\frac{2 g_{j}^{2}}{g_{i}^{2}-g_{j}^{2}} S_{i}^{z} S_{j}^{z}\right] \\
& i=1 \ldots L .
\end{aligned}
$$

These satisfy $\left[H, Q_{i}\right]=0$ and $\left[Q_{i}, Q_{j}\right]=0, \forall i, j=1 \ldots L$. Interpreting $H$ as $Q_{0}$, the conserved charges are bilinear in the spin operators and the number of linearly independent conserved charges exactly equals the number of spins in the system $(L+1)$. The system is thus Richardson-Gaudin integrable $[24,38]$. An additional conserved charge that helps establish integrability is given by

$$
\tilde{Q}=-2 S_{0}^{z} \sum_{i=1}^{L} g_{i}^{2} S_{i}^{z}+\frac{1}{2} \sum_{i, j=1}^{L} g_{i} g_{j}\left(S_{i}^{+} S_{j}^{-}+S_{i}^{-} S_{j}^{+}\right),
$$

which can be obtained as a linear combination of the conserved charges, $\tilde{Q}=\sum_{i=1}^{L} g_{i}^{2} Q_{i}$. The charge $\tilde{Q}$ relates directly to the square of the Hamiltonian

$$
\tilde{Q}=H^{2}-\omega_{0}^{2} / 4
$$

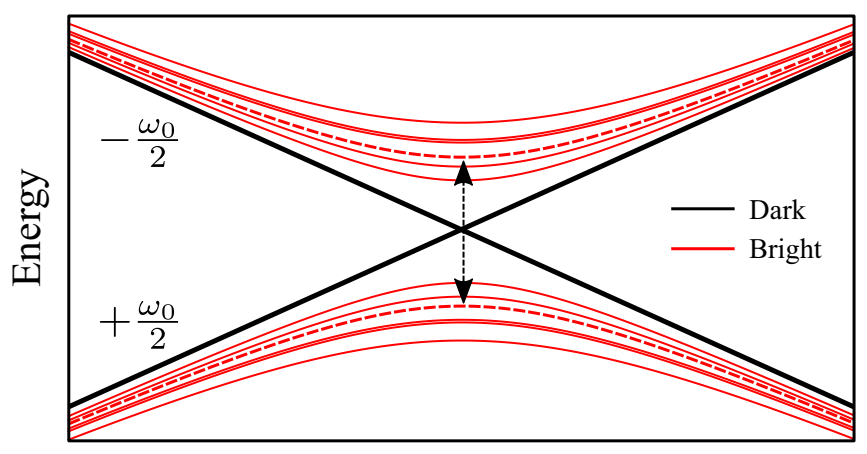

$\omega_{0}$

FIG. 2. Schematic representation of the energy spectrum as a function of the central field $\omega_{0}$, exhibiting highly degenerate dark states (black lines) and bands of bright states (red lines). The dashed lines highlight an example pair of bright states exhibiting level repulsion near resonance $\omega_{0}=\omega=0$. using the spin- $\frac{1}{2}$ properties of the central spin. Such quadratic relations between conserved charges are prevalent in Richardson-Gaudin systems [39-43].

Supersymmetry. The conserved charge $\tilde{Q}$ is the Hamiltonian of a $\mathcal{N}=2$ supersymmetric (SUSY) and integrable quantum theory [44-48], as it can be rewritten as

$$
\tilde{Q}=\mathcal{G}^{+} \mathcal{G}^{-}+\mathcal{G}^{-} \mathcal{G}^{+}, \quad\left(\mathcal{G}^{ \pm}\right)^{2}=0,
$$

with $\mathcal{G}^{ \pm}=\sum_{i} g_{i} S_{i}^{ \pm} S_{0}^{\mp}$ Hermitian conjugate supercharges satisfying $\left[S_{0}^{z}, \mathcal{G}^{ \pm}\right]=\mp \mathcal{G}^{ \pm}$. The eigenstates of $\tilde{Q}$ can be chosen to have fixed central spin magnetization $\left\langle S_{0}^{z}\right\rangle= \pm 1 / 2$ since $\left[\tilde{Q}, S_{0}^{z}\right]=0$. The supersymmetry imposes a specific structure on the eigenspectrum of $\tilde{Q}$. The (degenerate) ground-state manifold has eigenvalue zero and is spanned by singlet states annihilated by both $\mathcal{G}^{+}$and $\mathcal{G}^{-}$. States with a nonzero eigenvalue are twofold degenerate and arise as so-called superpartners $\left|\psi_{-}\right\rangle=|\downarrow\rangle_{0} \otimes\left|\mathcal{B}^{-}\right\rangle$and $\left|\psi_{+}\right\rangle=|\uparrow\rangle_{0} \otimes\left|\mathcal{B}^{+}\right\rangle$, satisfying $\mathcal{G}^{ \pm}\left|\psi_{ \pm}\right\rangle \propto\left|\psi_{\mp}\right\rangle$ and $\mathcal{G}^{\mp}\left|\psi_{ \pm}\right\rangle=0$.

While the eigenstates of $\tilde{Q}$ at finite (nonzero) energy are not necessarily eigenstates of $H$, the zero-energy eigenstates of $\tilde{Q}$ are guaranteed to be eigenstates of $H$ with eigenvalue $\pm \omega_{0} / 2$. It is precisely these zero modes that are the dark states. The remaining states in the spectrum of $H$ will arise as pairs of bright states, since the interaction term $\propto\left(\mathcal{G}+\mathcal{G}^{\dagger}\right)$ only couples superpartners: Two degenerate eigenstates of $\tilde{Q}$ with a nonzero eigenvalue $\Delta^{2}$ will split into nondegenerate states with energies $\pm \sqrt{\omega_{0}^{2} / 4+\Delta^{2}}$ in the spectrum of $H$ (see Fig. 2).

Dark and bright states. All eigenstates of $H$ can be expressed in terms of generalized spin raising operators acting on a vacuum state. To distinguish dark and bright states, we assume $S^{z}<0$ and take the vacuum to be the state with all spins maximally down [49]. Consider the Bethe states with $N$ spin excitations on top of the vacuum state $|0\rangle=|\downarrow\rangle_{0} \otimes_{i=1}^{L}$ $\left|-s_{i}\right\rangle$,

$$
\left|\psi\left(v_{1}, v_{2}, \ldots, v_{N}\right)\right\rangle=G^{+}\left(v_{1}\right) G^{+}\left(v_{2}\right) \cdots G^{+}\left(v_{N}\right)|0\rangle,
$$

with generalized spin raising operators that depend on (possibly complex) parameters $v_{1}, v_{2}, \ldots, v_{N}$ as

$$
G^{+}(v)=\sum_{i=1}^{L} \frac{g_{i}}{1-g_{i}^{2} v} S_{i}^{+} .
$$

As no spin raising operators act on the central spin, the central spin points along $-z$ in these states.

In the regime $S^{z} \leqslant 0$, the only allowed dark states are those with central spin down, which are exactly of the form of Eq. (7). Namely, dark states satisfy

$$
H|\mathcal{D}\rangle=-\frac{\omega_{0}}{2}|\mathcal{D}\rangle, \quad|\mathcal{D}\rangle=\left|\psi\left(v_{1}, v_{2}, \ldots, v_{N}\right)\right\rangle,
$$

provided the rapidities $v_{1} \ldots v_{N}$ satisfy the Bethe equations

$$
\sum_{i=1}^{L} \frac{s_{i} g_{i}^{2}}{1-g_{i}^{2} v_{a}}-\sum_{b \neq a}^{N} \frac{1}{v_{b}-v_{a}}=0
$$

for $a=1 \ldots N$. Importantly, these rapidities, and hence the structure of dark states, only depend on $\left\{g_{i}\right\}$ and not on $\omega_{0}$.

The bright states, on the other hand, can be written as a linear combination of two Bethe states of the form (7), with 


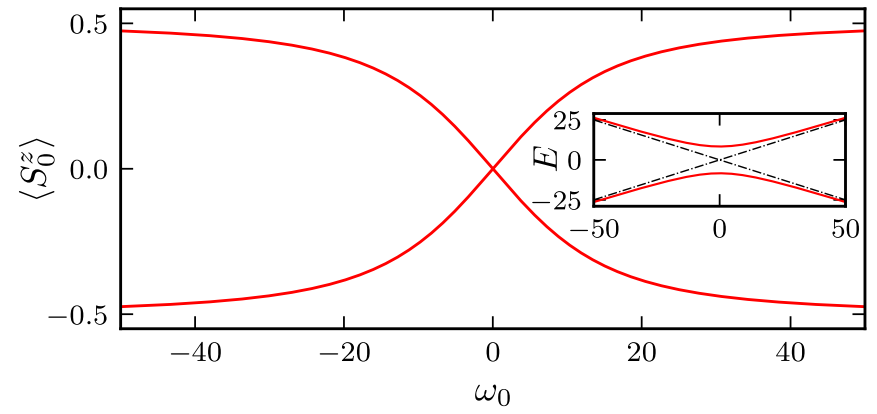

FIG. 3. Energy $E$ and central spin expectation value $\left\langle S_{0}^{z}\right\rangle$ for the pair of bright states corresponding to the ground state and the highest excited state for a central spin system with $L=100$ and $N=L / 4$, where $\left(g_{i}\right)^{-2}=i$ and $s_{i}=1 / 2, \forall i=1 \ldots L$. Dashed lines denote $E= \pm \omega_{0} / 2 . \sum_{i} 2 s_{i}\left(g_{i}\right)^{2}-\sum_{a} 2 / v_{a} \approx 69.9787$, and Bethe equations were solved using the methods developed in Ref. [40].

central spin down and up, respectively,

$$
\begin{aligned}
\mid \phi(E & \left.\left.; v_{1}, v_{2}, \ldots, v_{N-1}\right)\right\rangle \\
= & \left|\psi\left(0, v_{1}, v_{2}, \ldots, v_{N-1}\right)\right\rangle \\
& +\left(E+\frac{\omega_{0}}{2}\right) S_{0}^{+}\left|\psi\left(v_{1}, v_{2}, \ldots, v_{N-1}\right)\right\rangle \\
= & {\left[G^{+}(0)+\left(E+\frac{\omega_{0}}{2}\right) S_{0}^{+}\right]\left|\psi\left(v_{1}, v_{2}, \ldots, v_{N-1}\right)\right\rangle, }
\end{aligned}
$$

with rapidities now satisfying

$$
\begin{gathered}
E^{2}=\frac{\omega_{0}^{2}}{4}+\left(\sum_{i=1}^{L} 2 s_{i} g_{i}^{2}-\sum_{a=1}^{N-1} \frac{2}{v_{a}}\right), \\
1+v_{a} \sum_{i=1}^{L} \frac{s_{i} g_{i}^{2}}{1-g_{i}^{2} v_{a}}-v_{a} \sum_{b \neq a}^{N-1} \frac{1}{v_{b}-v_{a}}=0,
\end{gathered}
$$

for $a=1 \ldots N-1$. The bright states satisfy

$$
H|\mathcal{B}\rangle=E|\mathcal{B}\rangle, \quad|\mathcal{B}\rangle=\left|\phi\left(E ; v_{1}, \ldots, v_{N-1}\right)\right\rangle .
$$

While the rapidities $v_{1}, \ldots, v_{N-1}$ do not depend on $\omega_{0}$, the quadratic equation for the energy $E$ does. As such, each solution for $v_{1} \ldots v_{N-1}$ leads to two possible solutions for $E$ exhibiting level repulsion.

Interestingly, combining the Hellmann-Feynman theorem with Eq. (12) returns

$$
\left\langle S_{0}^{z}\right\rangle=\left\langle\frac{\partial H}{\partial \omega_{0}}\right\rangle=\frac{\partial E}{\partial \omega_{0}}=\frac{1}{4} \frac{\omega_{0}}{E},
$$

allowing the energy of any eigenstate to be uniquely related to its central spin polarization, as illustrated in Fig. 3 for a pair of bright states. Three limits are clearly reproduced: At resonance $\left(\omega_{0}=0\right)$ the central spin polarization vanishes exactly, and for $\omega_{0} \rightarrow \pm \infty$ the central spin is polarized as $\pm 1 / 2$. For dark states, $E= \pm \omega_{0} / 2$ returns the expected value of $\left\langle S_{0}^{z}\right\rangle= \pm 1 / 2$, such that this relation holds for all eigenstates.

We end this section with a few comments. For $S^{z}<0$, the solutions to Eqs. (10) and (13) respectively identify dark states in which the central spin points along $-z$, and bright states. For $S^{z}=0$, the Bethe equations (10) do not admit any solutions, and consequently all the states in the spectrum are bright. More generally, the Bethe equations (10) do not admit any solutions if $S^{z} \geqslant 0$. Nevertheless, the spectrum for $S^{z}>0$ has bright and dark states, with the central spin along $+z$ in the dark states [50]. The dark states now arise as additional solutions to the Bethe equations in Eq. (13) in which some of the rapidities are zero. In this regime, dark state energies are not given by $E$, but by $+\omega_{0} / 2$.

Explicit derivation. The structure of the conserved charges can be understood starting from $\tilde{Q}$ rather than $H$. To this end, we rewrite $\tilde{Q}$ as

$$
\tilde{Q}=\left(G^{-} G^{+}\right)\left(\frac{1}{2}+S_{0}^{z}\right)+\left(G^{+} G^{-}\right)\left(\frac{1}{2}-S_{0}^{z}\right) .
$$

Above, $G^{ \pm} \equiv G^{ \pm}(0)=\sum_{i=1}^{L} g_{i} S_{i}^{ \pm}$, and $\left(1 / 2 \pm S_{0}^{z}\right)$ projects the central spin along the $z$ direction. Hamiltonians of the form $G^{ \pm} G^{\mp}$ arise in the study of topological superconductivity and superfluidity [51-53], neutron pairing [54], and SachdevYe-Kitaev-like models [55]. These are Richardson-Gaudin integrable, and all results for their eigenstates and conserved charges can be found in, e.g., Refs. [24,25,38,55-58].

The conserved charges of $G^{-} G^{+}$are known to be

$$
\begin{aligned}
Q_{i}^{(-+)}= & S_{i}^{-} S_{i}^{+}+2 \sum_{j \neq i}^{L} \frac{g_{j}^{2}}{g_{i}^{2}-g_{j}^{2}} S_{i}^{z} S_{j}^{z} \\
& +\sum_{j \neq i}^{L} \frac{g_{i} g_{j}}{g_{i}^{2}-g_{j}^{2}}\left(S_{i}^{+} S_{j}^{-}+S_{i}^{-} S_{j}^{+}\right),
\end{aligned}
$$

which mutually commute and commute with $G^{-} G^{+}$. The conserved charges $Q_{i}^{(+-)}$of $G^{+} G^{-}$immediately follow from those of $G^{-} G^{+}$through spin inversion symmetry, since mapping $S^{ \pm} \rightarrow S^{\mp}$ and $S^{z} \rightarrow-S^{z}$ leaves the su(2) algebra invariant and maps $G^{-} G^{+} \rightarrow G^{+} G^{-}$. The conserved quantities of $\tilde{Q}$ then follow by combining these with the appropriate projection operators from Eq. (16) as

$$
Q_{i}=Q_{i}^{(-+)}\left(\frac{1}{2}+S_{0}^{z}\right)+Q_{i}^{(+-)}\left(\frac{1}{2}-S_{0}^{z}\right) .
$$

Using the spin- $\frac{1}{2}$ properties of the central spin, Eq. (18) simplifies to Eq. (3). It can be checked that, not only do these conserved charges satisfy $\left[Q_{i}, Q_{j}\right]=0, \forall i, j$, but they also satisfy $\left[H, Q_{i}\right]=0, \forall i$.

The eigenstates of $H$ can be similarly derived from the connection with the eigenstates of $\tilde{Q}$. The eigenstates of $G^{+} G^{-}$can be written as two kinds of Bethe states of the form (7), depending on the number of zero rapidities (see, e.g., Ref. [55]). The first kind are zero-energy (singlet) eigenstates where all rapidities are nonzero and satisfy Eq. (10),

$$
G^{+} G^{-}\left|\psi\left(v_{1}, \ldots, v_{N}\right)\right\rangle=0 .
$$

The action of $\tilde{Q}$ reduces to the action of $G^{+} G^{-}$on these states because $S_{0}^{z}=-1 / 2$ in the vacuum state in Eq. (7). As such, the zero-energy eigenstates of $G^{+} G^{-}$with central spin down are zero-energy eigenstates of $\tilde{Q}$, and are thus the dark states $|\mathcal{D}\rangle$. The condition $G^{-}|\mathcal{D}\rangle=0$ (2) follows directly from Eq. (19) since $G^{+} G^{-}$is positive definite. The zero modes of $\tilde{Q}$ with central spin up and their properties are similarly obtained from the zero modes of $G^{-} G^{+}$. 
The second kind of eigenstates of $G^{+} G^{-}$have one zero rapidity and nonzero positive eigenvalues,

$$
\begin{aligned}
G^{+} G^{-} & \left|\psi\left(0, v_{1}, \ldots, v_{N-1}\right)\right\rangle \\
= & \left(\sum_{i=1}^{L} 2 s_{i} g_{i}^{2}-\sum_{a=1}^{N-1} \frac{2}{v_{a}}\right)\left|\psi\left(0, v_{1}, \ldots, v_{N-1}\right)\right\rangle,
\end{aligned}
$$

where the nonzero rapidities satisfy Eq. (13). Furthermore, the state $\left|\psi\left(v_{1}, \ldots, v_{N-1}\right)\right\rangle$ is an eigenstate of $G^{-} G^{+}$with the same eigenvalue as in Eq. (20). Both states can be made into degenerate eigenstates of $\tilde{Q}$ by applying the proper central spin projectors and $\tilde{Q}$ has doubly degenerate eigenstates

$$
\begin{aligned}
& \left|\psi_{-}\right\rangle=G^{+}(0)\left|\psi\left(v_{1}, \ldots, v_{N-1}\right)\right\rangle, \\
& \left|\psi_{+}\right\rangle=S_{0}^{+}\left|\psi\left(v_{1}, \ldots, v_{N-1}\right)\right\rangle,
\end{aligned}
$$

with the same number of spin excitations $N$. These are related through $G^{ \pm} S_{0}^{\mp}\left|\psi_{ \pm}\right\rangle \propto\left|\psi_{\mp}\right\rangle$, such that $H$ only couples these states to each other. The eigenvalue equation for $H$ in this two-dimensional space leads to the quadratic equation (12) for the states (11). In the language of SUSY, the doublet states constituting bright states are coupled through $\mathcal{G}^{ \pm}$. The dark states are clearly annihilated by $\mathcal{G}^{+}=G^{+} S_{0}^{-}$since they have central spin down by construction and by $\mathcal{G}^{-}=G^{-} S_{0}^{+}$since they are annihilated by $G^{-}$, leading to singlet states.

The degenerate pairs of states (21) are eigenstates for each separate conserved charge $Q_{i}$. This follows from the observation that

$$
Q_{i}^{(+-)} G^{+}(0)=G^{+}(0) Q_{i}^{(-+)},
$$

such that if $\left|\psi\left(v_{1}, \ldots, v_{N-1}\right)\right\rangle$ is an eigenstate of $Q_{i}^{(-+)}$, then $G^{+}(0)\left|\psi\left(v_{1}, \ldots, v_{N-1}\right)\right\rangle$ is an eigenstate of $Q_{i}^{(+-)}$with the same eigenvalue. Their eigenvalues follow from the known eigenvalues of the conserved charges of either $G^{+} G^{-}$or $G^{-} G^{+}$(e.g., Ref. [55]). The eigenvalue $q_{i}$ of $Q_{i}$ for the dark states is given by

$$
q_{i}=2 s_{i}\left(\sum_{a=1}^{N} \frac{1}{1-g_{i}^{2} v_{a}}+\sum_{\substack{j=1 \\ j \neq i}}^{L} \frac{s_{j} g_{j}^{2}}{g_{i}^{2}-g_{j}^{2}}\right),
$$

and for the bright states by

$$
q_{i}=2 s_{i}\left(1+\sum_{a=1}^{N-1} \frac{1}{1-g_{i}^{2} v_{a}}+\sum_{\substack{j=1 \\ j \neq i}}^{L} \frac{s_{j} g_{j}^{2}}{g_{i}^{2}-g_{j}^{2}}\right) .
$$

Counting of states. The number of dark states can be obtained either by using dimensionality arguments or by counting the number of solutions to the Bethe equations. We focus on a system with a bath of $L$ spin- $\frac{1}{2}$ particles and $S^{z}<0$; generalizations fall outside the scope of this Rapid Communication. Dark states with $N \leqslant L / 2$ spin excitations have central spin down and live within a $\left(\begin{array}{l}L \\ N\end{array}\right)$-dimensional subspace of the Hilbert space. By Eq. (2), the dark states are projected by $G^{-}$onto the zero vector of the $\left(\begin{array}{c}L \\ N-1\end{array}\right)$-dimensional subspace of $N-1$ spin excitations. The corresponding dark-state manifold thus consists of precisely those $\left(\begin{array}{l}L \\ N\end{array}\right)-\left(\begin{array}{c}L \\ N-1\end{array}\right)$ orthogonal states with no parallel projection. This is exactly the number of solutions to the Bethe equations (10) for dark states; the number of solutions to such equations in Richardson-Gaudin models is well studied in the literature [23,42,59]. A similar expression for the number of dark states was presented in Ref. [33].

For bright states, the number of distinct solutions to Eq. (13) is given by $\left(\begin{array}{c}L \\ N-1\end{array}\right)[58,60]$, and each solution for the set $v_{1}, v_{2}, \ldots, v_{N-1}$ leads to two possible solutions for $E$ in Eq. (12) and a pair of bright states. Combined, the total number of eigenstates is given by

$$
\left[\left(\begin{array}{l}
L \\
N
\end{array}\right)-\left(\begin{array}{c}
L \\
N-1
\end{array}\right)\right]+2\left(\begin{array}{c}
L \\
N-1
\end{array}\right)=\left(\begin{array}{c}
L+1 \\
N
\end{array}\right),
$$

returning the expected number of eigenstates in each $S^{z}$ sector following the completeness of the Bethe ansatz for spin- $\frac{1}{2}$ Richardson-Gaudin systems [42]. It follows from the binomial coefficients that the number of both dark and bright states grows exponentially with bath size $L$.

Discussion. We established a family of integrable Richardson-Gaudin central spin models with anisotropic XX interactions by deriving the full set of conserved charges and Bethe eigenstates. Such models arise routinely in physical dipolar systems under resonance conditions [16-22], where our results are expected to be applicable. The eigenstates can be divided in two classes, dark or bright, depending on their qubit-bath entanglement properties.

Dark states exhibit no qubit-bath entanglement and can be used to store qubit states for quantum memory. Reference [33] proposed a scheme to store (and retrieve) an arbitrary qubit state in the state of the bath using adiabatic passage in the XXX model with weak inhomogeneous couplings $g_{j}$. This scheme immediately generalizes to the XX model, even in the presence of strong inhomogeneities. Starting from a product state in which the central spin is in the desired qubit state and the environment in a dark state, the qubit state can be encoded in a superposition of dark and bright states [taking, e.g., large positive $\omega_{0}$ values in Eq. (11)]. Since the dark component of the wave function is independent of $\omega_{0}$, an adiabatic passage to large negative values of $\omega_{0}$ transfers the qubit state to the bath as

$$
\begin{aligned}
& \left(u|\downarrow\rangle_{0}+v|\uparrow\rangle_{0}\right) \otimes\left|\mathcal{D}^{-}\right\rangle \\
& =u|\downarrow\rangle_{0} \otimes\left|\mathcal{D}^{-}\right\rangle+v|\uparrow\rangle_{0} \otimes \sum_{\mathcal{B}^{+}}\left|\mathcal{B}^{+}\right\rangle\left\langle\mathcal{B}^{+} \mid \mathcal{D}^{-}\right\rangle \\
& \stackrel{\omega_{0} \rightarrow-\infty}{\longrightarrow}|\downarrow\rangle_{0} \otimes\left[u\left|\mathcal{D}^{-}\right\rangle+v G^{+} \sum_{\mathcal{B}^{+}} e^{i \phi_{\mathcal{B}}}\left|\mathcal{B}^{+}\right\rangle\left\langle\mathcal{B}^{+} \mid \mathcal{D}^{-}\right\rangle\right],
\end{aligned}
$$

where $\phi_{\mathcal{B}}$ are the relative phases accrued during the passage. The final bath state serves as a robust memory for the qubit state; the qubit state can be retrieved by symmetrically reversing the process and accounting for the phases $\phi_{\mathcal{B}}$. This invites quantum memory applications in defect center or dot systems well described by the XX model.

Dark states are also known to limit hyperpolarization protocols which use a central qubit to transfer polarization to/from a bath [15]. In such protocols, the qubit is repeatedly polarized and manipulated (e.g., by tuning the field $\omega_{0}$ ) to induce qubit-bath exchange interactions. Since qubit-bath 
polarization exchanges are only possible in bright states, the bath polarization saturates to a value determined by the populated dark states. Several experiments have found saturation at high (above 60\%) spin bath polarizations [61-63], and strategies to overcome the limitations imposed by dark states have been proposed $[34,35]$. The explicit structure of dark states presented here may allow for the development of other hyperpolarization protocols with high saturation values of the bath polarization.

Given that the system is integrable, all eigenstates are nonthermal, not just the dark states. The bright states exhibit highly nonthermal expectation values, e.g., $\left\langle S_{0}^{z}\right\rangle=0$ at resonance (15), where the central spin is maximally entangled with the bath. The effect on the dynamics of $\left\langle S_{0}^{z}(t)\right\rangle$ is particularly pronounced. Considering, e.g., a system at resonance with an initially polarized state $\left\langle\psi(t=0)\left|S_{0}^{z}\right| \psi(t=0)\right\rangle=$ $1 / 2$, the long-time average of the observable $\overline{\left\langle S_{0}^{z}(t)\right\rangle}$ follows as

$$
\overline{\left\langle S_{0}^{z}(t)\right\rangle}=\frac{1}{2} \sum_{\mathcal{D}}|\langle\psi(t=0) \mid \mathcal{D}\rangle|^{2} .
$$

All dynamics of $\overline{\left\langle S_{0}^{z}(t)\right\rangle}$ are due to the dephasing of the bright states, whereas the nonthermal steady-state value retaining memory of the initial polarization is the rms average of the overlaps of the initial state with the (exponential number of) dark states. This is consistent with the integrability of the XX model. Integrable models are known to exhibit nonergodic behavior at long times and long-lived correlations due to the presence of conservation laws [64-66]. For the XX model, our expression for the conserved quantities (3) suggests that not only $S_{0}^{z}$, but also observables such as $S_{0}^{z} S_{i}^{z}$, may not thermalize at long times.

The Bethe ansatz also allows for exact theoretical and numerical studies in system sizes beyond the reach of exact diagonalization [24,29,30,67-71], providing different avenues for the study of nonequilibrium dynamics in central spin models.

Acknowledgments. We thank Anatoli Polkovnikov and Stijn De Baerdemacker for useful discussions, and Emil Yuzbashyan for pointing out the connection between Eq. (5) and supersymmetry. P.W.C. gratefully acknowledges support from a Francqui Foundation Fellowship from the Belgian American Educational Foundation (BAEF), Boston University's Condensed Matter Theory Visitors program, and EPSRC Grant No. EP/P034616/1. A.C. acknowledges support from the Sloan Foundation through Sloan Research Fellowships. This work was supported by NSF DMR-1813499 (T.V.), and NSF DMR-1752759 (T.V. and A.C.).
[1] R. Kosloff, J. Chem. Phys. 150, 204105 (2019).

[2] C. P. Koch, J. Phys.: Condens. Matter 28, 213001 (2016).

[3] G. De Lange, T. Van Der Sar, M. Blok, Z.-H. Wang, V. Dobrovitski, and R. Hanson, Sci. Rep. 2, 382 (2012).

[4] J. Cai, A. Retzker, F. Jelezko, and M. B. Plenio, Nat. Phys. 9, 168 (2013).

[5] T. Villazon, A. Polkovnikov, and A. Chandran, Phys. Rev. A 100, 012126 (2019).

[6] L. Dong, H. Liang, C.-K. Duan, Y. Wang, Z. Li, X. Rong, and J. Du, Phys. Rev. A 99, 013426 (2019).

[7] M.-H. Yung, J. Phys. B: At., Mol. Opt. Phys. 44, 135504 (2011).

[8] M. C. Tran and J. M. Taylor, arXiv:1801.04006.

[9] A. O. Sushkov, I. Lovchinsky, N. Chisholm, R. L. Walsworth, H. Park, and M. D. Lukin, Phys. Rev. Lett. 113, 197601 (2014).

[10] W.-B. He, S. Chesi, H.-Q. Lin, and X.-W. Guan, Phys. Rev. B 99, 174308 (2019).

[11] I. Schwartz, J. Scheuer, B. Tratzmiller, S. Müller, Q. Chen, I. Dhand, Z.-Y. Wang, C. Müller, B. Naydenov, F. Jelezko et al., Sci. Adv. 4, eaat8978 (2018).

[12] P. London, J. Scheuer, J.-M. Cai, I. Schwarz, A. Retzker, M. B. Plenio, M. Katagiri, T. Teraji, S. Koizumi, J. Isoya et al., Phys. Rev. Lett. 111, 067601 (2013).

[13] R. Hanson, L. P. Kouwenhoven, J. R. Petta, S. Tarucha, and L. M. K. Vandersypen, Rev. Mod. Phys. 79, 1217 (2007).

[14] J. Schliemann, A. Khaetskii, and D. Loss, J. Phys.: Condens. Matter 15, R1809 (2003).

[15] B. Urbaszek, X. Marie, T. Amand, O. Krebs, P. Voisin, P. Maletinsky, A. Högele, and A. Imamoglu, Rev. Mod. Phys. 85, 79 (2013).

[16] S. Hartmann and E. Hahn, Phys. Rev. 128, 2042 (1962).
[17] D. Rovnyak, Concepts Magn. Reson., Part A 32, 254 (2008).

[18] D. D. B. Rao, A. Ghosh, D. Gelbwaser-Klimovsky, N. Bar-Gill, and G. Kurizki, arXiv:1912.00613.

[19] C. W. Lai, P. Maletinsky, A. Badolato, and A. Imamoglu, Phys. Rev. Lett. 96, 167403 (2006).

[20] W. Ding, A. Shi, J. Q. You, and W. Zhang, Phys. Rev. B 90, 235421 (2014).

[21] J. M. Taylor, C. M. Marcus, and M. D. Lukin, Phys. Rev. Lett. 90, 206803 (2003).

[22] P. Fernández-Acebal, O. Rosolio, J. Scheuer, C. Müller, S. Müller, S. Schmitt, L. P. McGuinness, I. Schwarz, Q. Chen, A. Retzker et al., Nano Lett. 18, 1882 (2018).

[23] M. Gaudin, The Bethe Wavefunction (Cambridge University Press, Cambridge, UK, 2014).

[24] J. Dukelsky, S. Pittel, and G. Sierra, Rev. Mod. Phys. 76, 643 (2004).

[25] S. M. A. Rombouts, J. Dukelsky, and G. Ortiz, Phys. Rev. B 82, 224510 (2010).

[26] M. Bortz and J. Stolze, Phys. Rev. B 76, 014304 (2007).

[27] A. Faribault, P. Calabrese, and J.-S. Caux, J. Stat. Mech. (2009) P03018.

[28] M. Bortz, S. Eggert, C. Schneider, R. Stübner, and J. Stolze, Phys. Rev. B 82, 161308(R) (2010).

[29] A. Faribault and D. Schuricht, Phys. Rev. Lett. 110, 040405 (2013).

[30] P. W. Claeys, S. De Baerdemacker, O. E. Araby, and J.-S. Caux, Phys. Rev. Lett. 121, 080401 (2018).

[31] M. A. Jivulescu, E. Ferraro, A. Napoli, and A. Messina, Phys. Scr. T135, 014049 (2009).

[32] M. Jivulescu, E. Ferraro, A. Napoli, and A. Messina, Rep. Math. Phys. 64, 315 (2009). 
[33] J. M. Taylor, A. Imamoglu, and M. D. Lukin, Phys. Rev. Lett. 91, 246802 (2003).

[34] A. Imamoglu, E. Knill, L. Tian, and P. Zoller, Phys. Rev. Lett. 91, 017402 (2003).

[35] H. Christ, J. Cirac, and G. Giedke, Solid State Sci. 11, 965 (2009).

[36] C. Belthangady, N. Bar-Gill, L. M. Pham, K. Arai, D. Le Sage, P. Cappellaro, and R. L. Walsworth, Phys. Rev. Lett. 110, 157601 (2013).

[37] Z. Kurucz, M. W. Sørensen, J. M. Taylor, M. D. Lukin, and M. Fleischhauer, Phys. Rev. Lett. 103, 010502 (2009).

[38] G. Ortiz, R. Somma, J. Dukelsky, and S. Rombouts, Nucl. Phys. B 707, 421 (2005).

[39] P. W. Claeys, S. De Baerdemacker, and D. Van Neck, SciPost Phys. 3, 028 (2017).

[40] P. W. Claeys, S. De Baerdemacker, M. Van Raemdonck, and D. Van Neck, Phys. Rev. B 91, 155102 (2015).

[41] A. Faribault, O. El Araby, C. Sträter, and V. Gritsev, Phys. Rev. B 83, 235124 (2011).

[42] J. Links, SciPost Phys. 3, 007 (2017).

[43] An additional quadratic relation can be found as $\left(S^{z}\right)^{2}=\frac{1}{4} I+$ $C-\sum_{i=1}^{L} Q_{i}$, where $C$ is a linear combination of su(2) Casimir operators.

[44] P. Fendley, K. Schoutens, and J. de Boer, Phys. Rev. Lett. 90, 120402 (2003).

[45] X. Yang and P. Fendley, J. Phys. A: Math. Gen. 37, 8937 (2004).

[46] L. Huijse, A supersymmetric model for lattice fermions, Ph.D. thesis, University of Amsterdam, 2010.

[47] C. Hagendorf, J. Stat. Phys. 150, 609 (2013).

[48] C. Matsui, Nucl. Phys. B 913, 15 (2016).

[49] Any eigenstate can be constructed in two ways, either by expressing the state in terms of generalized spin raising operators acting on a vacuum state with all spins maximally down, or in terms of spin lowering operators acting on a dual vacuum state with all spins maximally up. For $S^{z}<0$, the former is more transparent and highlights the differences between bright states and dark states (with central spin necessarily down), and for $S^{z}>0$ the latter is more convenient since all dark states then have central spin up.

[50] Within the literature on integrability, this distinction is also referred to as "solutions above/below the equator" (see, e.g., Refs. [72-74]).

[51] M. Ibañez, J. Links, G. Sierra, and S.-Y. Zhao, Phys. Rev. B 79, 180501(R) (2009).
[52] S. Lerma H., S. M. A. Rombouts, J. Dukelsky, and G. Ortiz, Phys. Rev. B 84, 100503(R) (2011).

[53] G. Ortiz, J. Dukelsky, E. Cobanera, C. Esebbag, and C. Beenakker, Phys. Rev. Lett. 113, 267002 (2014).

[54] D. J. Dean and M. Hjorth-Jensen, Rev. Mod. Phys. 75, 607 (2003).

[55] E. Iyoda, H. Katsura, and T. Sagawa, Phys. Rev. D 98, 086020 (2018).

[56] J. Dukelsky, C. Esebbag, and P. Schuck, Phys. Rev. Lett. 87, 066403 (2001).

[57] M. Van Raemdonck, S. De Baerdemacker, and D. Van Neck, Phys. Rev. B 89, 155136 (2014).

[58] J. Links, I. Marquette, and A. Moghaddam, J. Phys. A: Math. Theor. 48, 374001 (2015).

[59] A. Faribault and H. Tschirhart, SciPost Phys. 3, 009 (2017).

[60] Y. Shen, P. S. Isaac, and J. Links, Nucl. Phys. B 937, 28 (2018).

[61] A. S. Bracker, E. A. Stinaff, D. Gammon, M. E. Ware, J. G. Tischler, A. Shabaev, A. L. Efros, D. Park, D. Gershoni, V. L. Korenev, and I. A. Merkulov, Phys. Rev. Lett. 94, 047402 (2005).

[62] B. Urbaszek, P.-F. Braun, T. Amand, O. Krebs, T. Belhadj, A. Lemaítre, P. Voisin, and X. Marie, Phys. Rev. B 76, 201301(R) (2007).

[63] E. A. Chekhovich, M. N. Makhonin, K. V. Kavokin, A. B. Krysa, M. S. Skolnick, and A. I. Tartakovskii, Phys. Rev. Lett. 104, 066804 (2010).

[64] E. T. Jaynes, Phys. Rev. 106, 620 (1957).

[65] M. Rigol, V. Dunjko, V. Yurovsky, and M. Olshanii, Phys. Rev. Lett. 98, 050405 (2007).

[66] L. Vidmar and M. Rigol, J. Stat. Mech. (2016) 064007.

[67] A. Faribault, P. Calabrese, and J.-S. Caux, Phys. Rev. B 77, 064503 (2008).

[68] M. S. Foster, M. Dzero, V. Gurarie, and E. A. Yuzbashyan, Phys. Rev. B 88, 104511 (2013).

[69] O. El Araby and D. Baeriswyl, Phys. Rev. B 89, 134521 (2014).

[70] A. J. A. James, R. M. Konik, P. Lecheminant, N. J. Robinson, and A. M. Tsvelik, Rep. Prog. Phys. 81, 046002 (2018).

[71] N. J. Robinson, A. J. J. M. de Klerk, and J.-S. Caux, arXiv:1911.11101.

[72] G. P. Pronko and Y. G. Stroganov, J. Phys. A: Math. Gen. 32, 2333 (1999).

[73] C. Korff, J. Phys. A: Math. Gen. 38, 47 (2004).

[74] R. J. Baxter, J. Stat. Phys. 108, 1 (2002). 